\title{
Heating of a particulate by radio-frequency electric and magnetic fields
}

\author{
Herman Bosman, Wilkin Tang, Y. Y. Lau, ${ }^{\text {a) }}$ and R. M. Gilgenbach \\ Department of Nuclear Engineering and Radiological Sciences, University of Michigan, \\ Ann Arbor, Michigan 48109-2104
}

(Received 17 June 2004; accepted 19 August 2004)

\begin{abstract}
With the use of a highly symmetrical model, the heating of a spherical particulate by a predominantly radio-frequency electric field and by a predominantly rf magnetic field is solved exactly using the Maxwell equations. It is found that, in general, heating by the rf magnetic field is dominant whenever $\delta<a$, where $\delta$ is the resistive skin depth and $a$ is the radius of the particulate, which may either be nonmagnetic or magnetic. The known analytic scaling laws in the various regimes are recovered, from the static case to very high frequency, subject to $\lambda \gg a$, where $\lambda$ is the free space wavelength of the $\mathrm{rf}$ field. The analysis may form a theoretical basis in the heating phenomenology of particulates. (C) 2004 American Institute of Physics. [DOI: 10.1063/1.1806269]
\end{abstract}

Heating of a particulate is fundamental to the heating phenomenology in physical, chemical, and biological sciences. When exposed to an electromagnetic wave, the finite electrical conductivity in the particulate leads to ohmic dissipation via the electric field within the particulate. This electric field is often nonuniform. While its physical origin may come directly from the external electric field, it may also come from the time-varying or rf magnetic field which, through Faraday's law, would produce an inductive electric field within this particulate. In an electromagnetic wave, the rf electric and magnetic fields are strongly coupled, and it may be questionable whether one is justified to consider the heating by the rf electric field and by the rf magnetic field separately and independently, in particular when both components must be simultaneously interacting with the particulate.

This distinction between heating by the rf electric field and by the rf magnetic field is natural in the low-frequency (quasistatic) limit, following for example the approach of Landau and Lifshitz. ${ }^{1}$ It may also be recognized in the highfrequency regime, if the particulate is placed in the region of strong rf electric field or in the region of strong rf magnetic field of a resonant microwave cavity. In fact, there has recently been strong evidence that heating by the rf magnetic field in a resonant cavity may be dominant, in both industrial heating ${ }^{2}$ and in the melting of debris that reside on the cavity surface where there is a strong rf magnetic field. ${ }^{3,4}$

In this letter, we seek to resolve the degree of ohmic heating, via the rf magnetic field or via the rf electric field, in terms of the particulate size, its electrical conductivity, and the wavelength of the electromagnetic radiation to which it is exposed. Our analysis is valid from the static (dc) limit to high frequency. We place a homogeneous, spherical particulate of radius $a$ at the center of a spherical cavity. The cavity has radius $b$ and its wall is infinitely conducting (Fig. 1). In the absence of this particulate, the spherical cavity admits the fundamental transverse electric $\mathrm{TE}_{110}$ mode, which has a maximum rf electric field (and a null rf magnetic field) at the center, as well as the transverse magnetic $\mathrm{TM}_{110}$ mode, which has a maximum rf magnetic field (and a null rf electric field) at the center (Fig. 1). Both the TE mode and TM mode

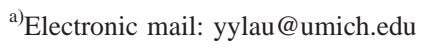

have an infinite quality factor $Q$ since the cavity wall is lossless.

When a small lossy particulate is inserted at the center of this cavity (Fig. 1), the modes are slightly damped and the quality factors become finite. This change in the quality factor in the TE (TM) mode immediately gives the degree of ohmic dissipation that is due to the rf electric (magnetic) field. The advantage of this simple model is that the high degree of symmetry allows exact solution of the full set of Maxwell equations, completely accounting for the inductive electric field, the displacement current, and the conduction current. Extremely accurate calculations of the eigenmode's complex frequency (whose imaginary part, the damping rate, is proportional to $1 / Q$ ) can be obtained when $a \ll b$. The well-known quasistatic results may also be recovered by letting $b$ tend to infinity, in which case the eigenfrequencies of both the $\mathrm{TE}_{110}$ and $\mathrm{TM}_{110}$ mode tend to zero.

For the fundamental TM (TE) mode in the spherical cavity, the rf electric (magnetic) field has only a $\phi$-component (Fig. 1), which may be written in the general form as $[A j(\xi)+B y(\xi)] \sin \theta$. In this expression, $A$ and $B$ are arbitrary constants; $\xi=k r$, where $k=\omega(\varepsilon \mu)^{1 / 2}$ is the wave number in the region whose (complex) permittivity and permeability are, respectively, $\varepsilon$ and $\mu$, and $j(\xi)=\xi^{-2} \sin \xi-\xi^{-1} \cos \xi$ and $y(\xi)=-\xi^{-2} \cos \xi-\xi^{-1} \sin \xi$ are the spherical Bessel functions of order 1. All other components of the rf fields, and the exact dispersion relation for the eigenfrequencies, may be obtained by using Maxwell's equations, and demanding that the rf solutions are regular at $r=0$, that the tangential rf electric field is zero at $r=b$, and that the tangential rf electric
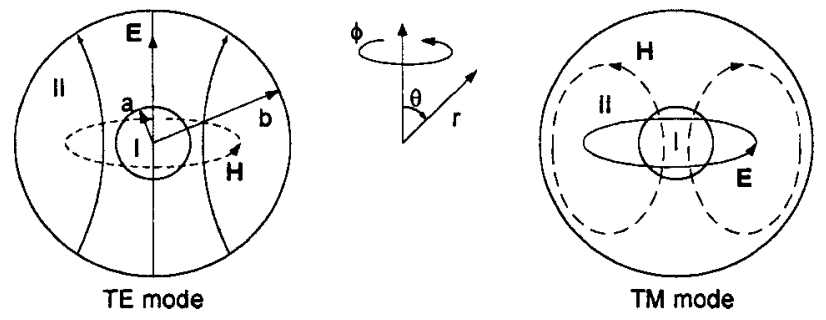

FIG. 1. The geometry, and the rf field patterns for the $\mathrm{TE}_{110}$ mode (left) and the $\mathrm{TM}_{110}$ mode (right). The mode index (110) refers to variations in $r, \theta$, and $\phi$. 
field $(\mathbf{E})$ and the tangential rf magnetic field $(\mathbf{H})$ are continuous at $r=a$ (Fig. 1).

We will use the subscripts 1 and 2 throughout to denote the respective values in region I $(r<a)$ and region II $(a<r<b)$ (Fig. 1). Note that if region II is a vacuum, $\varepsilon_{2}$ $=\varepsilon_{0}$ and $\mu_{2}=\mu_{0}$; we shall focus mainly on this case. We further assume that $\mu_{1}$ is real, and that $\varepsilon_{1}=\varepsilon_{1 r}+\sigma / \mathrm{j} \omega$ is complex if there is a finite electrical conductivity $\sigma$ in the particulate. Note that this formulation covers the rf heating of a particulate that is either magnetic (large $\mu_{1} / \mu_{0}$ ) or electric $\left(\mu_{1} / \mu_{0}=1\right)$ through either the rf electric field (TE mode) or the rf magnetic field (TM mode).

In the absence of the spherical particulate ( $a=0$ limit), the well-known eigenfrequencies $\omega_{\mathrm{E}}$ and $\omega_{\mathrm{M}}$ for the $\mathrm{TE}_{110}$ and $\mathrm{TM}_{110}$ vacuum modes in a spherical cavity ${ }^{5}$ are given by $\eta_{\mathrm{E}}=2.74371$ and $\eta_{\mathrm{M}}=4.4934$, respectively, with $\eta_{\mathrm{E}, \mathrm{M}}$ $=\omega_{\mathrm{E}, \mathrm{M}} b / c$, where $c$ is the speed of light in vacuum.

The presence of a small, lossy particulate leads to a small damping rate $\gamma_{\mathrm{E}}\left(\gamma_{\mathrm{M}}\right)$ of the TE (TM) mode. Under the assumption $b \gg a$, that is, the free space wavelength $\lambda$ $=2 \pi c / \omega \gg a$, where $\omega=\omega_{\mathrm{E}}$ or $\omega_{\mathrm{M}}$, a careful expansion of the exact dispersion relation, to be published elsewhere, gives the following damping rates for the TE and TM modes:

$$
\frac{\gamma_{E}}{\omega_{E}}=\frac{Y^{\prime}\left(\eta_{E}\right)}{\eta_{E} J^{\prime \prime}\left(\eta_{E}\right)} \frac{J\left(\xi_{2 E}\right)}{Y\left(\xi_{2 E}\right)}\left[\frac{Y^{\prime}\left(\xi_{2 E}\right)}{\xi_{2 E} Y\left(\xi_{2 E}\right)}-\frac{J^{\prime}\left(\xi_{2 E}\right)}{\xi_{2 E} J\left(\xi_{2 E}\right)}\right] \operatorname{Im}\left(\frac{1}{Z_{E}}\right),
$$

$$
\begin{aligned}
Z_{E}= & \left(\frac{\mu_{1}}{\mu_{0}}\right) \frac{J^{\prime}\left(\xi_{1}\right)}{\xi_{1} J\left(\xi_{1}\right)}-\frac{Y^{\prime}\left(\xi_{2 E}\right)}{\xi_{2 E} Y\left(\xi_{2 E}\right)}, \\
\frac{\gamma_{M}}{\omega_{M}}= & \frac{y\left(\eta_{M}\right)}{\eta_{M} j^{\prime}\left(\eta_{M}\right)} \frac{J^{\prime}\left(\xi_{2 M}\right)}{Y^{\prime}\left(\xi_{2 M}\right)}\left[\frac{Y\left(\xi_{2 M}\right)}{\xi_{2 M} Y^{\prime}\left(\xi_{2 M}\right)}\right. \\
& \left.-\frac{J\left(\xi_{2 M}\right)}{\xi_{2 M} J^{\prime}\left(\xi_{2 M}\right)}\right] \operatorname{Im}\left(\frac{1}{Z_{M}}\right), \\
Z_{M}= & \left(\frac{\mu_{1}}{\mu_{0}}\right) \frac{J\left(\xi_{1}\right)}{\xi_{1} J^{\prime}\left(\xi_{1}\right)}-\frac{Y\left(\xi_{2 M}\right)}{\xi_{2 M} Y^{\prime}\left(\xi_{2 M}\right)} .
\end{aligned}
$$

In Eqs. (1)-(4), $J(\xi)=\xi j(\xi), Y(\xi)=\xi y(\xi)$, and a prime denotes the derivative with respect to the argument. In the arguments, $\quad \xi_{2 \mathrm{E}}=\omega_{\mathrm{E}} a / c=\eta_{\mathrm{E}}(a / b), \quad \xi_{2 \mathrm{M}}=\omega_{\mathrm{M}} a / c=\eta_{\mathrm{M}}(a / b)$, which are both real; $\xi_{1}=\omega\left(\varepsilon_{1} \mu_{1}\right)^{1 / 2} a$ in which $\mu_{1}$ is real, $\varepsilon_{1}$ $=\varepsilon_{1 r}+\sigma / \mathrm{j} \omega$ is complex, with $\omega=\omega_{\mathrm{E}}\left(\omega_{\mathrm{M}}\right)$ for the TE (TM) mode. The normalized damping rates, Eqs. (1) and (3), may then be immediately determined once the following four dimensionless parameters are given: $a / \lambda, \delta / a, \mu_{1} / \mu_{0}$, and $\varepsilon_{1 r} / \varepsilon_{0}$. In this letter, the skin depth $\delta=\left(2 / \omega \mu_{0} \sigma\right)^{1 / 2}$ is always defined with respect to the vacuum permeability $\mu_{0}$. We should stress that the only assumption that has been made to derive Eqs. (1)-(4) is that $a \ll \lambda$, that is, $a \ll b$. There is no restriction on the relative size $\delta / a$. That is, the ratio $\sigma / \omega \varepsilon_{1 r}$ may assume an arbitrary value between zero and infinity, a rather nontrivial generalization for the evaluation of the ohmic loss in the particulate. Thus, the de limit is also governed by Eqs. (1)-(4).

The damping rate $\gamma$ given in Eqs. (1) or (3) is related to the average power $P$ that is absorbed by the particulate of the TE (or TM) mode, and therefore to the quality factor $Q$ of that mode of the (loaded) cavity through the well-known relation, $P=2 \gamma U=\omega U / Q$, where $U$ is the average electro-

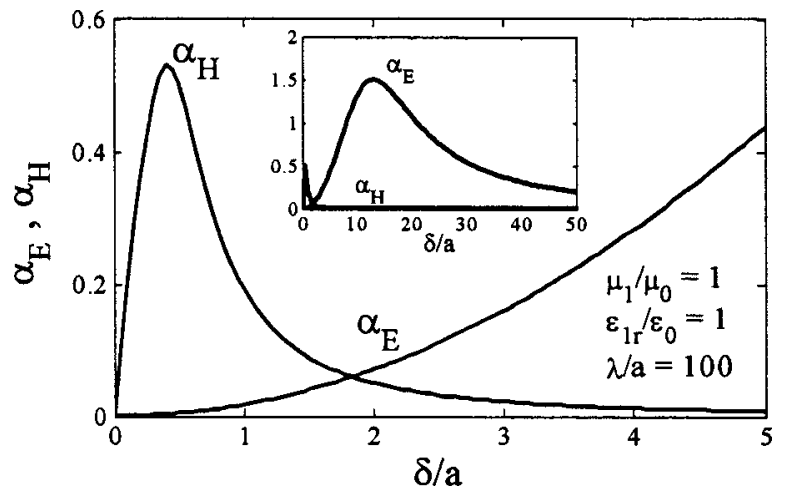

FIG. 2. The polarizabilities, $\alpha_{\mathrm{E}}$ and $\alpha_{\mathrm{H}}$, as a function of $\delta / a$ for $\mu_{1} / \mu_{0}$ $=1, \varepsilon_{1 r} / \varepsilon_{0}=1$, and $\lambda / a=100$.

magnetic energy stored in the empty cavity in that TE (or TM) mode (Ref. 5, p. 258). Note that once we calculate the damping rate $\gamma$ (or $Q$ ) of the mode, we immediately know the power dissipated within the entire particulate without explicitly calculating the detailed field distribution within that particulate. In fact, it was this observation that motivated us to undertake a general study over the huge parameter space in the manner presented in this letter.

To facilitate comparison with the literature, ${ }^{1,6}$ we express the power dissipated in the particulate in terms of the dimensionless polarizabilities, $\alpha_{\mathrm{E}}$ and $\alpha_{\mathrm{H}}$, defined by

$$
\begin{aligned}
& P=\alpha_{E} \omega_{E}\left(\frac{1}{2} \varepsilon_{0} E_{0}^{2}\right) V_{a},(\mathbf{T E}) \\
& P=\alpha_{H} \omega_{M}\left(\frac{1}{2} \mu_{0} H_{0}^{2}\right) V_{a},(\mathbf{T M}) .
\end{aligned}
$$

In Eq. (5), $E_{0}\left(H_{0}\right)$ is the peak rf electric (magnetic) field of the TE (TM) mode at the center of the cavity in the absence of the particulate, whose volume is $V_{a}=(4 \pi / 3) a^{3}$. The $\alpha$ in Eq. (5) is proportional to the $\gamma / \omega$ given by Eqs. (1) and (3), and the proportionality constant depends on the TE or TM mode.

The polarizability versus the normalized skin depth $(\delta / a)$ with $\mu_{1} / \mu_{0}=1, \varepsilon_{1 r} / \varepsilon_{0}=1$, and $\lambda / a=100$ for the TE and TM mode is shown in Fig. 2. It can be seen from Fig. 2 that the polarizability for the TM mode $\left(\alpha_{\mathrm{H}}\right)$ dominates for small $\delta / a$. For large $\delta / a$, the polarizability for the TE mode $\left(\alpha_{\mathrm{E}}\right)$ surpasses that of the TM mode. The inset of Fig. 2 shows $\alpha_{\mathrm{E}}$ and $\alpha_{\mathrm{H}}$ on a much larger scale. Since the polarizability is directly proportional to the power absorbed, for small $\delta / a$ the power absorbed by the particulate when it is exposed to an electromagnetic wave is, in general, primarily through the rf magnetic field, whereas for large $\delta / a$, the power absorbed is primarily through the rf electric field. For very large $\delta / a$, both $\alpha_{\mathrm{E}}$ and $\alpha_{\mathrm{H}}$ tend to zero, as expected of an almost nonconducting particulate.

The asymptotic dependence shown in Fig. 2 is given by

$$
\begin{gathered}
\alpha_{H} \cong \frac{9}{4}\left(\frac{\delta}{a}\right), \quad \delta / a \ll 1 ; \\
\alpha_{H} \cong \frac{1}{5}\left(\frac{a}{\delta}\right)^{2}, \quad \delta / a \gg 1,
\end{gathered}
$$



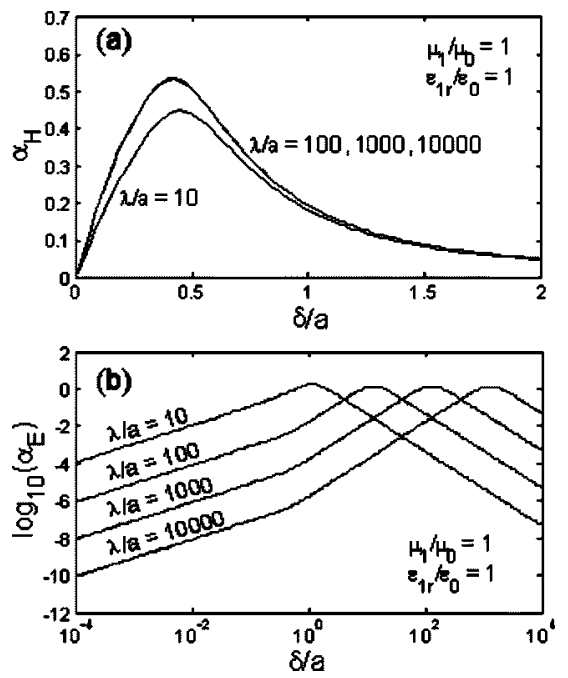

FIG. 3. The polarizabilities $\alpha_{\mathrm{H}}$ (a), and $\alpha_{\mathrm{E}}$ (b), as a function of $\delta / a$ for $\mu_{1} / \mu_{0}=1, \varepsilon_{1 r} / \varepsilon_{0}=1$, and various values of $\lambda / a$.

$$
\alpha_{E} \cong \begin{cases}9 \pi^{2}\left(a \delta / \lambda^{2}\right), & \delta / a \ll 1, \\ 18 \pi^{2}(\delta / \lambda)^{2}, & 1 \ll \delta / a \ll \lambda / a, \\ (\lambda / \delta)^{2} /\left(2 \pi^{2}\right), & 1 \ll \lambda / a \ll \delta / a .\end{cases}
$$

Equations (6a) and (6b) are the same as those in Landau and Lifshitz. ${ }^{1}$ Equations (7a) and (7b), which, respectively, show the linear and quadratic dependence of $\alpha_{\mathrm{E}}$ on $\delta$, as shown in Fig. 2 for $\delta / a<5$, were presented in Bosman et al. ${ }^{4}$ derived from different approaches. ${ }^{7}$ Equation (7c), which shows the decay of $\alpha_{\mathrm{E}}$ for very large values of $\delta$, as shown in the inset of Fig. 2, is easily derived from a quasistatic analysis.

The scalings shown in Eqs. (6) and (7) were confirmed in Fig. 3, obtained for different ratios of $\lambda / a$ with $\mu_{1} / \mu_{0}$ $=1, \varepsilon_{1 r} / \varepsilon_{0}=1$. The insensitivity of $\alpha_{\mathrm{H}}$ to $\lambda / a$, for large values of $\lambda / a$, is apparent in Fig. 3(a) and Eq. (6). Figure 3(b) shows the dependence of $\alpha_{\mathrm{E}}$, which is reflected in Eqs. (7a)-(7c). In Fig. 3(b), $\delta / a \sim \mathrm{O}(1)$ marks the transition from Eq. (7a) to (7b), whereas the maxima of $\alpha_{\mathrm{E}}$, which occur approximately when $\sigma=\omega \varepsilon_{1 r}$, mark the transition from Eq. (7b) to (7c). Once more, an examination of the scales in Fig. 3 shows that heating by $\mathrm{rf}$ magnetic field is the dominant mechanism if $\delta / a \ll 1$, thus offering a plausible interpretation of the observations reported in Refs. 2-4.

Pretending that the damping rate Eqs. (1) and (3) are applicable to a magnetic particulate with a high $\mu$, we plot $\alpha_{\mathrm{E}}$ and $\alpha_{\mathrm{H}}$ for different values of $\mu_{1} / \mu_{0}$ in Figs. 4(a) and 4(b), setting $\lambda / a=1000$, and $\varepsilon_{1 r} / \varepsilon_{0}=1$. The relative sensitivity of $\alpha_{\mathrm{H}}$ to $\mu_{1}$, and the insensitivity of $\alpha_{\mathrm{E}}$ to $\mu_{1}$, is noted. Once more, $\alpha_{\mathrm{H}}$ dominates over $\alpha_{\mathrm{E}}$ when $\delta / a \ll 1$.

For a small-sized particulate placed off-center of a microwave resonant cavity, the local electromagnetic field, at that position, may be considered as a superposition of a TE
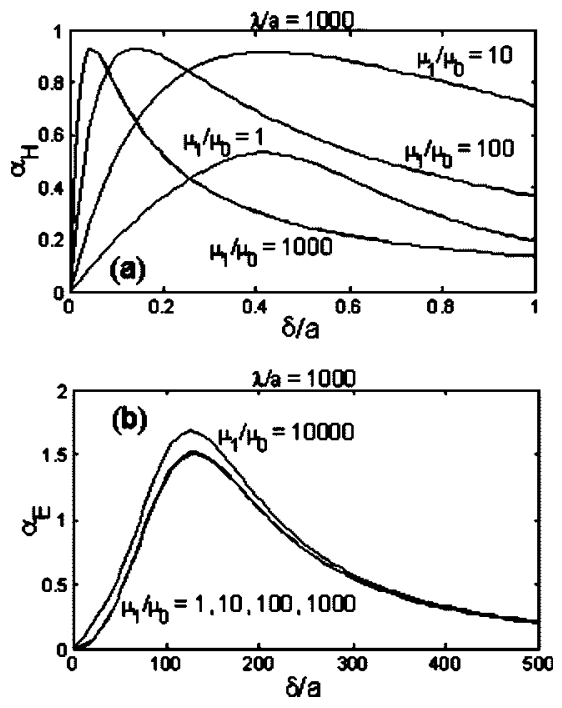

FIG. 4. The polarizabilities $\alpha_{\mathrm{H}}$ (a), and $\alpha_{\mathrm{E}}$ (b), as a function of $\delta / a$ for $\varepsilon_{1 r} / \varepsilon_{0}=1, \lambda / a=1000$, and various values of $\mu_{1} / \mu_{0}$.

mode and a TM mode, which we have individually treated very accurately. Our analysis then immediately gives a reliable indication of the degree of rf heating from such a decomposition, without having to perform the extremely difficult three-dimensional calculations of the perturbed electromagnetic fields in a highly nonsymmetrical geometry, over vastly different scales of $\lambda, \delta$, and $a$, as shown in Fig. 3 (b), for instance. As a further extension, the absorption from a collection of the particulates may also be readily assessed once the particulates' spatial distribution within the cavity is given.

The authors thank Bahman Hafizi, John Luginsland, David Chernin, Arne Fliflet, John Booske, and Jeff Calame for useful discussions on various aspects of this problem. This work was supported by DOE grant DE-FG0298ER54475, AFOSR, DUSD ( $\&$ \& T) under the Innovative Microwave Vacuum Electronics MURI Program, managed by the Air Force Office of Scientific Research under Grant F49620-99-1-0297. It was also funded through AFOSR by a USDoD MURI04 grant.

${ }^{1}$ L. D. Landau and E. M. Lifshitz, Electrodynamics of continuous media (Pergamon, New York, 1984), p. 322.

${ }^{2}$ J. Cheng, R. Roy, and D. Agrawal, J. Mater. Sci. Lett. 20, 1561 (2001).

${ }^{3}$ V. A. Dolgashev and S. G. Tantawi, AIP Conf. Proc. 691, 151 (2003).

${ }^{4}$ H. Bosman, Y. Y. Lau, and R. M. Gilgenbach, AIP Conf. Proc. 691, 234 (2003).

${ }^{5}$ S. Ramo, J. R. Whinnery, and T. Van Duzer, Fields and waves in communications electronics (Wiley, New York, 1994), p. 508.

${ }^{6}$ G. L. Carr, S. Perkowitz, and D. B. Tanner, in Infrared and MM waves, edited by K. J. Budden, (Academic, New York, 1985), Vol. 13, p. 171.

${ }^{7}$ H. Bosman, Ph.D thesis, University of Michigan, Ann Arbor, 2004. 